\title{
Uma análise dos tipos de jogadores em uma plataforma de gamificação incorporada a um sistema juiz on-line
}

\author{
Marcela Pessoa $^{1,2}$, Rafaela Melo ${ }^{1}$, Gabriel Haydar ${ }^{1}$, David B. F. de Oliveira ${ }^{1}$, \\ Leandro S. G. Carvalho ${ }^{1}$, Elaine H. T. de Oliveira ${ }^{1}$, Tayana Conte ${ }^{1}$, \\ Filipe Dwan Pereira $^{1,3}$, Luiz Rodrigues ${ }^{4}$, Seiji Isotani ${ }^{4}$ \\ ${ }^{1}$ Programa de Pós-Graduação em Informática (PPGI) \\ Instituto de Computação - Universidade Federal do Amazonas (UFAM) \\ Manaus - AM - Brasil \\ ${ }^{2}$ Escola Superior de Tecnologia - Universidade do Estado do Amazonas (EST/UEA) \\ Manaus - AM - Brasil \\ ${ }^{3}$ Departamento de Ciência da Computação - Universidade Federal de Roraima (UFRR) \\ Boa Vista - RR - Brasil \\ ${ }^{4}$ Instituto de Ciências Matemáticas e da Computação - Universidade de São Paulo (USP) \\ São Carlos - SP - Brasil \\ \{msppessoa,rmelo,gigh, david, galvao, elaine, tayana\}@icomp.ufam.edu.br \\ filipe.dwan@ufrr.br, \{lalrodrigues, sisotani\}@usp.br
}

\begin{abstract}
Research seeks to understand the preferences of different user profiles in games and gamified environments. In this sense, this paper aims to identify and analyze the preferences and behaviors of users of different profiles within a gamification platform created around a multiplayer RPG game, developed and implemented in real introductory programming classes. User profiles were identified using Hexad taxonomy, and user interactions of each type with the platform's game elements were stored in log records. Through an analysis of the students' logs, it was noticed that each profile has nuances in their preferences and behaviors, which are discussed in this study. It is believed that the results of this research can provide important insights for the development and improvement of personalized gamified environments.
\end{abstract}

Resumo. Pesquisas buscam entender o comportamento de diferentes perfis de usuários em jogos e ambientes gamificados. Nesse sentido, o objetivo deste artigo é identificar e analisar as preferências e comportamentos dos usuários de diferentes perfis dentro de uma plataforma de gamificação criada em torno de um jogo RPG multiplayer, desenvolvido e implantado em turmas reais de introdução à programação. Os perfis dos usuários foram identificados por meio da taxonomia Hexad, e as interações dos usuários de cada tipo com os elementos de jogos da plataforma foram armazenadas em registros de logs. Através de uma análise dos logs dos alunos, percebeu-se que cada perfil possui diferenças em seus comportamentos, que são discutidas neste estudo. Acredita-se que os resultados desta pesquisa trazem insights importantes para o desenvolvimento e aprimoramento de ambientes gamificados personalizados. 


\section{Introdução}

$\mathrm{O}$ uso de juízes on-line como ferramentas de apoio à aprendizagem de programação tem crescido ao longo dos últimos anos [Galvão et al. 2016, Bez et al. 2014, Oyibo and Vassileva 2019, Pereira et al. 2020]. Juízes on-line são sistemas capazes de avaliar a corretude de códigos-fonte de forma automatizada e em tempo real, e entre as vantagens do uso de tais sistemas pode-se citar: diminuição do tempo de correção dos códigos por parte dos professores, avaliações mais consistentes, e aumento do número de problemas resolvidos pelos alunos [Kurnia et al. 2001]. Apesar das vantagens, o aprendizado de programação continua sendo uma atividade complexa e pouco motivante para muitos alunos [Qian and Lehman 2017]. Por isso, tem crescido o interesse por estratégias capazes de trazer ludicidade aos juízes on-line, na esperança de despertar o engajamento e a motivação dos estudantes. Uma das estratégias mais estudadas para esse fim é a utilização de jogos ou a incorporação de elementos de jogos nos sistemas juízes on-line [Bez et al. 2014, Kasahara et al. 2019, Petit et al. 2017, Sinly et al. 2018].

Nesse sentido, em Pessoa et al. (2019) é proposta a integração de uma plataforma de gamificação baseada em um jogo RPG multiplayer com um sistema juiz on-line denominado CodeBench. Entretanto, segundo os mesmos autores, os resultados obtidos após a integração indicaram que a plataforma de gamificação incentivou somente os alunos que já tinham alta probabilidade de aprovação. Essa observação abriu espaço para mais estudos sobre o impacto do uso de jogos e ambientes gamificados para despertar o interesse e a motivação dos usuários.

Estudos indicam que existem diferentes perfis de usuários de jogos e/ou ambientes gamificados, e que, para maximizar o número de usuários engajados e motivados, é necessário considerar as características pessoais de cada um deles [Jia et al. 2016, Hassan et al. 2019, Tondello et al. 2019a, Lopez and Tucker 2019, Klock et al. 2020, Tondello et al. 2016]. Alinhada a essa descoberta, a literatura científica apresenta diferentes propostas de taxonomias de usuários de jogos e sistemas gamificados, que procuram identificar os perfis considerando as motivações individuais com que esses usuários desempenham cada ação dentro dos ambientes [Bartle 1996, Nacke et al. 2014, Marczewski 2015]. Uma vez que os perfis sejam identificados, é possível maximizar o engajamento desses usuários através da implementação de elementos de jogos alinhados às preferências e motivações dos perfis identificados [Rodrigues et al. 2020].

Embora existam meios para identificar os perfis dos usuários, ainda não existe um consenso sobre as suas preferências. Posto isso, este trabalho tem como objetivo identificar e analisar as preferências, comportamentos e interesses de cada tipo de jogador de um ambiente gamificado. Vale ressaltar que esse conhecimento é valioso para os projetistas de sistemas gamificados, pois sem ele é muito difícil desenvolver sistemas que sejam realmente capazes de motivar e engajar os diferentes tipos de usuários [Jia et al. 2016, Rodrigues et al. 2020]. Para possibilitar esse estudo, foram implementados novos elementos de jogos na plataforma CodePlay, por exemplo, missões, chat, doação de itens, entre outros, e definido um conjunto de 22 variáveis de estudos. Usando o framework Hexad, que é uma taxonomia para jogadores de ambientes gamificados proposta por [Marczewski 2015], os usuários da plataforma foram classificados em seis perfis: Filantropo, Socializador, Espírito Livre, Conquistador, Jogador e Disruptor. Através de uma análise das estatísticas de uso dos elementos por cada um dos seis perfis identifi- 
cados, este trabalho apresenta um breve estudo sobre quais são os elementos de jogos que mais despertam o interesse de cada perfil de usuário.

Este artigo está assim organizado: a Seção 2 apresenta os aspectos teóricos sobre perfis de usuário; a Seção 3 descreve os trabalhos relacionados; na Seção 4 são apresentados os elementos de jogos incluídos na gamificação; na Seção 5 são registrados os resultados alcançados; e por fim, na Seção 6 as conclusões e trabalhos futuros.

\section{Referencial Teórico}

As pessoas costumam ter preferências diferentes entre si, e por isso é necessário que os jogos levem em consideração essas diferenças [Hallifax et al. 2019]. Em gamificação, uma maneira de fazer isso é adaptando os elementos de jogos de acordo com os perfis dos usuários, que podem ser identificados através das taxonomias disponíveis na literatura [Bovermann and Bastiaens 2020, Bartle 1996]. As taxonomias estabelecem regras e fundamentos que podem ser usados para agrupar os usuários com características similares.

Neste trabalho está sendo utilizado o framework Hexad [Marczewski 2015], que é uma taxonomia baseada no perfil dos jogadores que agrupa os usuários de acordo com as características de suas interações com os elementos de jogos. O Hexad tem como fundamento a teoria da autodeterminação (STD), que estuda as razões e motivações que levam as pessoas a realizarem determinadas tarefas, que podem ser classificadas entre motivações intrínsecas ou motivações extrínsecas [Ryan and Deci 2000b]. Com base nessa teoria, o Hexad diferencia os usuários considerando as motivações individuais com que desempenham as ações no jogo. A motivação intrínseca está ligada ao desejo de realizar tarefas por pura satisfação interna, e a motivação extrínseca está ligada ao desejo de realizar as tarefas com o objetivo de obter compensações externas [Ryan and Deci 2000a].

Os usuários do Hexad são divididos em seis tipos [Marczewski 2015]: (i) Conquistadores, motivados por domínio, gostam de desafios e explorar coisas novas no jogo. São atraídos por elementos de jogos como troféus, missões, nível/progresso e batalha com chefão; (ii) Socializadores, motivados por relacionamento, por isso preferem interagir com os outros jogadores e criar vínculos sociais. Os elementos de jogos apreciados podem ser guilda, pressão de tempo, elementos de competição, redes e descobertas sociais; (iii) Filantropos, motivados por propósito e significado, gostam de ajudar os outros jogadores. São atraídos por elementos de jogos como doação de coletáveis, possibilidade de partilhar conhecimento ou cuidar de pessoas; (iv) Espíritos Livres, motivados por autonomia e podem ter dois perfis diferentes: o perfil "criador", que gosta de customizar os ambientes e avatares, ou o perfil "explorador", que prefere explorar caminhos alternativos e conteúdos desbloqueáveis; (v) Disruptores, motivados por mudanças, gostam de explorar os limites, as vezes pelo desejo de encontrar falhas no sistema. São atraídos por elementos de jogos como anonimato, atividades anarquistas, entretanto podem ajudar quando veem a possibilidade de serem ouvidos; e (vi) Jogadores, motivados por recompensa. Têm preferências por elementos como pontos, prêmios, emblemas, recompensas, jogos de azar e elementos de comparação, como os placares. Os quatro primeiros tipos são ligados às motivações intrínsecas e os outros dois tipos são ligados a fatores de motivação extrínseca. O Hexad possui um questionário [Tondello et al. 2016, Tondello et al. 2019b] composto por 24 itens, em escala Likert de sete pontos, que geram pontuações indicando o grau de pertencimento do usuário a cada um dos seis tipos de usuários. 


\section{Trabalhos Relacionados}

Alguns trabalhos têm pesquisado sobre o uso de jogos ou ambientes gamificados com o objetivo de despertar o engajamento de diferentes perfis de usuários durante a realização de atividades tipicamente não-lúdicas. Sinly et al. (2018) propuseram uma estratégia de gamificação para o sistema juiz on-line UMN OJ, que é usado para treinamento de competidores de programação na Universitas Multimedia Nusantara. Os elementos de jogos implementados foram placar, emblema e feedback, e os perfis dos jogadores foram identificados através da Taxonomia de Bartle [Bartle 1996]. Os autores avaliaram o sistema através do total de acessos dos alunos, que aumentou de $37 \%$, no sistema juiz on-line sem gamificação, para 95\%, com o juiz on-line gamificado. Foi aplicado o Hedonic Motivation System Adoption Model (HMSAM) [Lowry et al. 2012] para avaliar a aceitação do sistema, tendo obtido a aprovação da maioria dos participantes, mas não avaliaram a utilização segundo os perfis de Bartle.

Amado e Roleda (2020), em um sistema gamificado para aprendizagem de física, investigaram as preferências dos elementos de jogos e o engajamento dos estudantes considerando os perfis Hexad dos alunos. Os elementos de jogos foram escolhidos com base em Tondello et al. (2016). Segundo os autores, para os tipos Filantropo, Disruptor e Jogador, o elemento de jogo preferido é a escolha, implementada no curso por possibilitar que os alunos escolham se trabalham sozinhos ou em grupo e a dificuldade do desafio (fácil, normal ou difícil). Os tipos Espírito Livre, Socializador e Conquistador, preferem as recompensas na forma de pontos, conquistas e moedas. Os tipos Conquistador também mostraram preferência por coleção e comércio virtual (emblemas, moedas e power-ups) e os Socializadores por conteúdos desbloqueáveis. Quanto ao elemento de jogo com menor preferência, os alunos de diferentes tipos escolheram a competição, na forma de placar. Segundo os alunos, esse elemento gera sentimentos e emoções negativas provocados quando o aluno tem uma classificação baixa. Foi também avaliado o engajamento dos alunos no curso, medido com o Student Course Engagement Questionnaire (SCEQ) [Handelsman et al. 2005], que mede o envolvimento de estudantes universitários em determinados cursos. Nas médias gerais e nas médias por tipo Hexad, os Jogadores têm pontuação de média à alta, indicando que todos os alunos mostraram alto nível de envolvimento no curso, sugerindo que, para esse curso de física gamificado, o tipo de Jogador Hexad não tem influência no engajamento dos aluno.

Lopez e Tucker (2019) analisam a relação entre os tipos de usuários Hexad [Tondello et al. 2016] e o desempenho individual dos jogadores em um aplicativo que detecta os movimentos do corpo para concluir tarefas físicas. Os usuários foram divididos em grupo controle, que interagiu com a aplicação não gamificada, e grupo experimental, que interagiu com uma versão gamificada, contendo três elementos de jogos: pontos, conteúdo desbloqueável e avatar. Os participantes que interagiram com a gamificação tiveram melhor desempenho do que os outros participantes. Ao avaliar os tipos de usuários, os participantes que obtiveram pontuação alta no tipo Conquistador melhoraram seu desempenho mais do que os outros participantes. Os que obtiveram pontuação alta no tipo Filantropo ou Espírito Livre tiveram melhor desempenho que outros participantes. Os participantes que obtiveram pontuação alta no tipo Espírito Livre, Filantropo ou Jogador, tiveram desempenho pior no aplicativo gamificado em comparação com o não gamificado. Em análise da percepção identificada por meio de questionário, os elementos ponto e avatar foram percebidos como frustrantes e conteúdo desbloqueável como útil e preferido. 
Assim como os trabalhos aqui descritos, este artigo analisa a utilização dos elementos de jogos de acordo com os perfis de usuário, nesse caso usando a taxonomia Hexad, aplicada a uma gamificação, que contém um jogo de entretenimento, integrada a um sistema juiz on-line. Para isso, neste trabalho é proposta a inclusão de elementos de jogos que atendam às diferentes motivações descritas nos tipos de usuários, de forma a permitir que os alunos utilizem os elementos que mais os atraiam. Os dados de utilização da gamificação são armazenados, possibilitando a análise dos elementos mais usados por cada perfil de usuário. Uma vez que a literatura mostra que a gamificação não agrega valor a todos os usuários, a maior diferença entre a gamificação proposta e as pesquisas descritas é o grande número de elementos de jogos implementados durante este trabalho, que foi possível pela flexibilidade fornecida por um jogo de entretenimento do gênero RPG multiplayer, e tem potencial de oferecer uma boa experiência para todos os usuários.

\section{CodePlay}

O CodePlay é uma plataforma de gamificação incorporada ao sistema juiz on-line Codebench, desenvolvida em torno de um jogo RPG tradicional, contendo personagens, edificações e seres mágicos próprios. Ao solucionar um exercício de programação no juiz on-line, uma carta é sorteada para o aluno, definindo sua sorte e permitindo que ele avance na estória do jogo. Dentro do ambiente (mundo do jogo), os alunos podem interagir entre si (aspecto multiplayer), coletar recompensas, realizar missões, vencer inimigos e explorar vários elementos existentes no mapa. Assim como na maioria dos jogos RPG, os alunos podem escolher seus próprios avatares (feminino ou masculino) e acumular pontos de experiência e moedas à medida que evoluem no jogo [Pessoa et al. 2019].

Para analisar as preferências dos tipos de jogadores definidos no Hexad, foram implementados novos elementos de jogos no CodePlay, com base nas sugestões de Marczewski (2015) e considerando os seis perfis presentes no Hexad. Foram implementados pelo menos dois elementos para cada perfil de jogador, entretanto, para os perfis baseados em motivação intrínseca é oferecido uma maior quantidade de elementos de jogos. Alguns elementos de jogos propostos por Marczewski (2015) foram reconstruídos para que entrassem em harmonia com os elementos já presentes no jogo, como por exemplo, o enredo. $\mathrm{O}$ atual enredo acontece em um mundo virtual onde todos os alunos foram aprisionados. Esse mundo virtual é repleto de monstros e de muitos perigos, e foi criado por um ser chamado Marduk usando tecnologia computacional e linguagens de programação do mundo real. Como esse mundo foi criado usando linguagens de computador, estudar programação faz com que os alunos desenvolvam poderes dentro desse mundo. Quanto mais estudam programação, mais poderosos se tornam, e esse é o único meio de vencer Marduk e passar ileso de todos os perigos do mundo virtual.

Entre os elementos de jogos adicionados [Melo et al. 2021], destacam-se: (i) Doação de itens, armas e moedas: permite a doação de itens, armas e moedas entre alunos; (ii) Troca de mensagens: possibilita a troca de mensagens entre alunos; (iii) Itens de destaque de mérito: permite que os alunos colecionem emblemas que atestam conquistas relacionadas à disciplina. Os emblemas são de ouro, prata e bronze para os indicadores de média das avaliações, média das listas de exercícios e frequência de acesso ao juiz on-line; (iv) Barras de progresso: são indicações visuais que mostram o rendimento dos alunos nas disciplinas (nas listas de exercícios e avaliações), bem como o percentual dos lugares fechados e missões que já foram encontrados pelos usuários 
em cada fase do jogo; (v) Ranking com rating dos jogadores: os jogadores são divididos em três grupos, de acordo com o valor de experiência (XP) de cada um: Bronze, Prata ou Ouro. Os jogadores só veem os alunos de seu próprio grupo no ranking. Os alunos ganham XP à medida que resolvem os exercícios escolares propostos pelos professores; (vi) Missões (quests): a cada fase do jogo são disponibilizadas missões e, em algumas delas, o aluno precisa lutar contra monstros. Normalmente essas missões servem como uma maneira de quebrar a linearidade presente nos jogos, ou como uma maneira de abrir conteúdos exclusivos ou disponibilizar armas secretas; (vii) Lugares secretos: assim como as missões, todas as fases contêm lugares secretos, onde os alunos podem se deparar com outros personagens, encontrar moedas, XP e itens; (viii) Easter eggs: são dispostos dentro de lugares fechados e de forma aleatória nas fases a fim de manter a imprevisibilidade; e (iv) Opções anarquistas: ao longo do jogo os alunos encontram cenários onde podem escolher alternativas, entre elas opções anarquistas, como roubar uma receita ou liberar um monstro.

A versão atual do jogo é composta por sete fases e em todas elas tem edificações bloqueadas, cartas de experiência e moedas, missões coletivas, presentes na primeira versão do jogo, e são propostos novos elementos de jogos, como missões secundárias, passagens secretas, locais de descanso para recuperar a vida e batalha final de cada fase, havendo alteração dos objetivos, enredo, cenários e descrições dos eventos. Em algumas missões secundárias é dada a opção de escolhas anarquistas, como por exemplo: liberar um orc (personagem presente em contos de fantasia medieval) aprisionado, roubar uma receita, não avisar sobre uma simulada falha no jogo, entre outras. A Figura 1 apresenta algumas telas do jogo. Na Figura 1a é apresentada a missão secundária de encontrar a chave da dispensa e poder escolher se liberta ou não um orc. Caso o aluno opte por libertar o orc, os demais alunos precisarão se esforçar para aprisioná-lo novamente. Na Figura $1 \mathrm{~b}$ é apresentada a tela de envio de mensagem para outro aluno. Quando o avatar de um aluno se aproxima do avatar de outro, surge um balão de conversa que pode ser usado para enviar uma mensagem. Mesmo que o aluno destinatário não esteja on-line, a mensagem será entregue assim que ele entrar no jogo. É possível, também, enviar mensagem sem que o aluno esteja próximo. Além disso, o jogador tem acesso ao Diário de Missões que lista as missões disponíveis e conquistadas (Figura 1c). É importante destacar que não são registrados no Diários de Missões as passagens secretas e easter eggs já que eles têm a característica de serem procurados pelos jogadores. A cada povoado (que representa uma fase) conquistado, ou após o convite inicial para visitar as lojas de armas, poções e magias, o aluno pode se teleportar (Figura 1d).

\section{Cenário Experimental e Resultados}

Esta seção descreve o cenário experimental, as variáveis que compõem a base de dados, a análise dos tipos de jogadores e as discussões sobre a utilização dos elementos de jogos.

\subsection{Cenário Experimental}

Devido ao contexto pandêmico ${ }^{1}$, o sistema foi utilizado no período letivo de 2020/1, ocorrido no primeiro semestre de 2021 , de forma remota. Por ter sido um período atípico, foram considerados os alunos que interagiram com pelo menos um elemento de jogo da segunda fase, o que equivale a um mês de aula, nas disciplinas de Introdução à Programação

\footnotetext{
${ }^{1}$ Pandemia de Covid-19, que influenciou as atividades acadêmicas a partir do ano de 2020.
} 


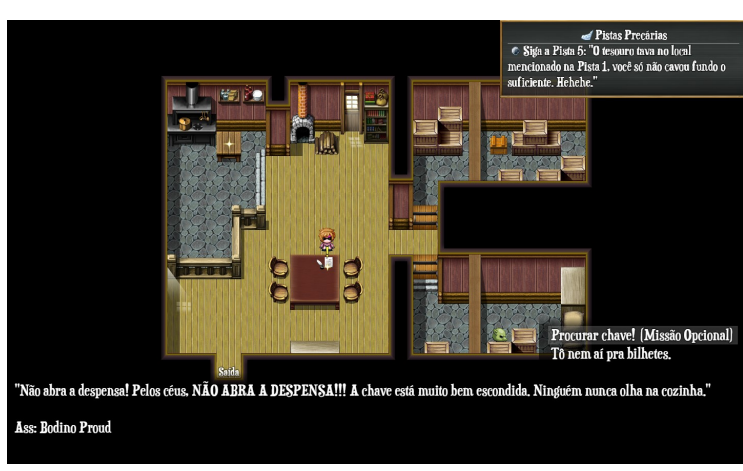

(a)

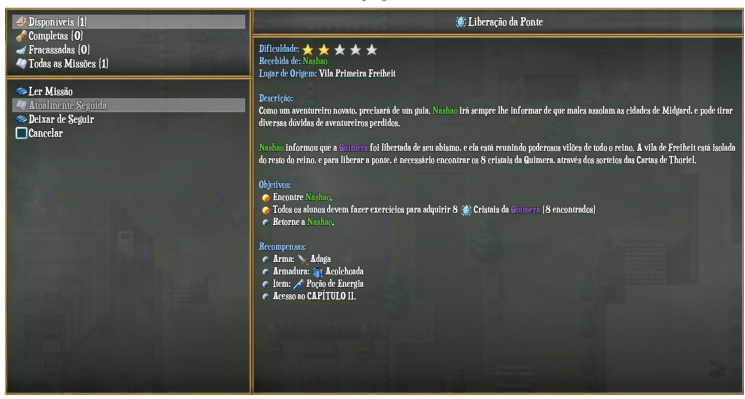

(c)

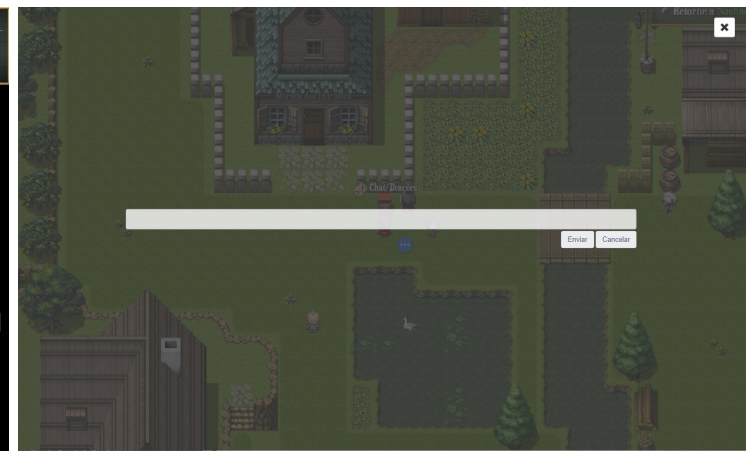

(b)

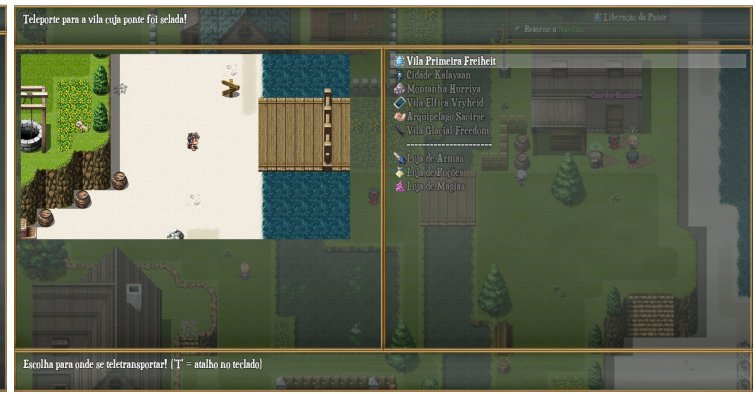

(d)

Figura 1. (a) Missão secundária com opção anarquista. (b) Envio de mensagens a outro aluno. (c) Diário de missões. (d) Teleporte do aluno.

de Computadores e Estruturas de Dados. Além disso, foi pedido aos alunos que respondessem o questionário de perfil de jogador Hexad [Tondello et al. 2016]. No total 95 alunos utilizaram o sistema e responderam o questionário. Desses, 72 alunos apresentaram um único perfil Hexad dominante, 16 alunos com dois perfis Hexad dominantes e sete apresentaram três perfis dominantes. Como o objetivo do trabalho é analisar o comportamento do usuário na gamificação e avaliar a diferença entre os perfis Hexad, analisando os elementos que foram efetivamente utilizados pelos alunos, foram considerados nos experimentos somente os 72 jogadores com um único perfil dominante, isto é, que obteve maior pontuação em um único perfil. Todas as interações dos alunos com o sistema foram registradas em um sistema de logs.

\subsection{Variáveis analisadas e criação da base de dados de estudo}

Para avaliar a utilização dos elementos de jogos, foram criadas 22 variáveis de estudo, extraídas dos $\log s$ de utilização da gamificação. As variáveis refletem a utilização dos elementos de jogos, são elas: total de acesso à (i) barra de progresso, (ii) ao diário, (iii) ao ranking, (iv) ao menu equipar, (v) ao menu itens, (vi) ao menu jogadores, (vii) ao menu outras opções; total de vezes que o jogador (viii) ligou o som, (ix) deixou o minimapa visível, (x) recebeu o reconhecimento através dos óculos; somatório de (xi) XP e (xii) moedas, total de (xii) interações realizadas com NPCs (non-player characters), mensagens (xiv) enviadas a colegas e (xv) recebidas; total de itens (xvi) doados e (xvii) recebidos, (xviii) missões secundárias e (xvix) passagens secretas concluídas, (xx) easter eggs encontrados, (xxi) escolhas anarquistas e (xxii) vezes que passou o mouse sobre o avatar de outro aluno para obter informações sobre o mesmo (infobox). A maioria das atividades é opcional, permitindo que os jogadores utilizem os elementos mais compatíveis com seus 
interesses e tendências pessoais. Essa flexibilidade é importante para possibilitar a análise dos perfis de uso de cada tipo de jogador Hexad, seguindo as suas preferências.

Para analisar a utilização dos elementos, foi construída uma base de dados onde cada registro contém os dados de um aluno. Cada registro (aluno) contém um total de 23 campos, representando as 22 variáveis descritas anteriormente, e um campo adicional representando o tipo de usuário Hexad do aluno. Como as variáveis possuem diferentes valores de grandezas (por exemplo, o total de pontos de experiência ou moedas acumuladas é muito maior quando comparado ao número de missões secretas), os dados foram normalizados para ficarem todos no intervalo entre zero e um.

\subsection{Tipos de jogadores}

Dos 72 alunos que se encaixaram nos critérios de inclusão discutidos na Seção 5.1, é possível observar na Figura 2 que o perfil com mais alunos é o Conquistador ( $\mathrm{n}=23$; $32 \%)$, seguido por Filantropo $(\mathrm{n}=17 ; 23 \%)$, Jogador $(\mathrm{n}=14 ; 19 \%)$, Espírito livre $(\mathrm{n}=$ $14 ; 19 \%)$ e Socializador $(\mathrm{n}=5 ; 7 \%)$. Nenhum aluno apresentou o perfil Disruptor.

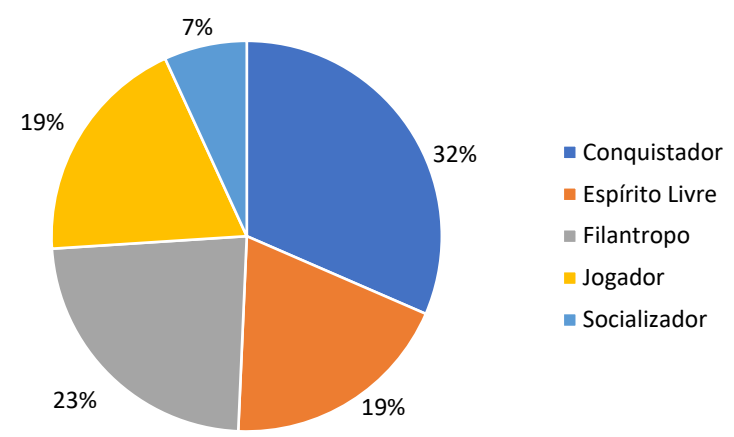

Figura 2. Distribuição dos jogadores agrupados de acordo com os perfis Hexad.

\subsection{Resultados e discussões}

Para a análise dos dados, os usuários foram agrupados de acordo com o seu perfil Hexad e foi calculada a média de utilização de cada uma das 22 variáveis descritas na Seção 5.2. As médias foram normalizadas para diminuir a diferença das grandezas, gerar o gráfico e possibilitar uma análise visual da utilização dos elementos de jogos. A Figura 3 apresenta o gráfico de utilização dos elementos por tipo de jogador. As linhas representam os 22 elementos e as colunas representam os cinco tipos Hexad encontrados nos dados. É possível observar que os cinco tipos de jogadores tiveram comportamentos diferentes.

O perfil Conquistador, por ser motivado por desafios e exploração, aspectos muito oferecidos no jogo, se apresentou com um perfil bem uniforme, utilizando a maioria dos elementos. As exceções foram as doações efetuadas e recebidas e as áreas secretas que foram menos exploradas. O perfil Espírito Livre, que é motivado por autonomia [Marczewski 2015], explorou bastante alguns elementos de jogos, não tendo acessado somente as áreas secretas e doou menos itens do que recebeu. Um fato a ser explorado é se o estilo do jogo por ser RPG e proporcionar mais liberdade para o jogador explorar o cenário, pode ter beneficiado esse perfil. Observa-se que esse tipo de jogador teve maior interação com os NPCs, que dão dicas e contam o enredo do jogo, fornecendo a sensação de exploração ao jogador. Além disso, eles foram atraídos pelas missões secundárias, pois só é possível cumprir tais missões através da exploração do ambiente. Entretanto, 
esperava-se maior utilização das áreas secretas, que foi o elemento não utilizado pelo perfil. Por outro lado, os espíritos livres acessaram mais o ranking, que é um elemento de competição, o que foi uma surpresa, pois se esperava isso do perfil Jogador.

O tipo Filantropo, tal como o Conquistador, se manteve bastante uniforme na utilização dos elementos de jogos, com destaque para easter eggs e ativar o som. Esperava-se que os Filantropos utilizassem mais a doação de itens, entretanto o elemento nem foi utilizado, o que sugere a necessidade de um estudo mais aprofundado sobre a forma como o elemento está implementado.

\begin{tabular}{|c|c|c|c|c|c|}
\hline & conquistador & espírito livre & filantropo & jogador & socializador \\
\hline moedas & 0,20 & 0,38 & 0,70 & 0,45 & 1,00 \\
\hline area_secreta & 0,04 & 0,00 & 0,00 & 1,00 & 0,00 \\
\hline acesso_ranking & 0,23 & 1,00 & 0,66 & 0,83 & 0,00 \\
\hline barra_progresso & 0,20 & 1,00 & 0,41 & 0,51 & 0,04 \\
\hline minimapa & 0,64 & 1,00 & 0,80 & 0,84 & 0,10 \\
\hline mensagens_enviadas & 0,09 & 0,49 & 0,36 & 1,00 & 0,08 \\
\hline mensagens_recebidas & 0,27 & 0,57 & 0,50 & 1,00 & 0,20 \\
\hline npc & 0,43 & 1,00 & 0,44 & 0,60 & 0,16 \\
\hline itens_doados & 0,00 & 0,17 & 0,14 & 1,00 & 0,00 \\
\hline doacoes_recebidas & 0,00 & 1,00 & 0,00 & 1,00 & 0,00 \\
\hline easter_egg & 0,65 & 0,84 & 1,00 & 0,84 & 0,00 \\
\hline acesso_diario & 0,34 & 1,00 & 0,62 & 0,54 & 0,00 \\
\hline acesso_itens & 0,76 & 1,00 & 0,65 & 0,83 & 0,07 \\
\hline acesso_equipar & 0,67 & 1,00 & 0,70 & 0,76 & 0,14 \\
\hline som & 0,92 & 0,93 & 1,00 & 0,91 & 0,57 \\
\hline acesso_jogadores & 0,35 & 1,00 & 0,84 & 0,48 & 0,00 \\
\hline experiencia & 0,91 & 1,00 & 0,70 & 0,96 & 0,25 \\
\hline outras_opcoes & 0,52 & 1,00 & 0,53 & 0,64 & 0,18 \\
\hline infobox & 0,69 & 0,72 & 0,63 & 1,00 & 0,40 \\
\hline oculos & 0,61 & 1,00 & 0,31 & 0,58 & 0,31 \\
\hline escolha_anarquista & 0,64 & 1,00 & 0,82 & 1,00 & 0,00 \\
\hline missoes_secundarias & 0,94 & 1,00 & 0,68 & 0,82 & 0,49 \\
\hline
\end{tabular}

Figura 3. Gráfico com os dados reais da utilização dos 22 elementos de jogos, de acordo com o perfil Hexad identificado nos alunos.

Para os alunos do tipo Jogador, apesar de gostarem de elementos de competição, observou-se menor número de acessos ao ranking, quando comparado ao tipo Espírito Livre. Entretanto, tais alunos tiveram maior acesso ao elemento infobox, que fornece informações sobre os outros jogadores ao passar o mouse sobre seus avatares. Os tipos Jogadores também mostram preferência por áreas secretas, pois como este tipo de jogador é motivado por recompensa, ele procura explorar o máximo de lugares possíveis em busca de prêmios. Um fato curioso é ser o tipo de usuário que mais utilizou mensagens, tanto envios quanto recebimentos, o que desperta o interesse em analisar o conteúdo das mensagens para verificar se estão relacionadas a aspectos de competição, como por exemplo, pedido de dicas dos demais alunos. Por fim, os Socializadores, apesar de serem motivados por relacionamento, fizeram pouco uso de mensagens. Por outro lado, é o perfil que mais usou o recurso de doações e mais coletou moedas, que também podem ser doadas.

\section{Conclusão e Trabalhos Futuros}

Neste trabalho foram implementados novos elementos de jogos a uma plataforma de gamificação criada em torno de um jogo RPG multiplayer, e em seguida foram feitas análises sobre o uso dos novos elementos por usuários de diferentes perfis. Os perfis foram identificados através da taxonomia Hexad, que tem sido bastante usada na literatura em contextos semelhantes. A plataforma de gamificação, integrada a um juiz on-line, foi utilizada em disciplinas práticas de programação da Universidade Federal do Amazonas. 
As interações dos alunos com a plataforma foram armazenadas através de um sistema de logs. A partir dos registros desses $\log s$, foi definido um conjunto de 22 variáveis representando o uso dos elementos pelos usuários da plataforma. Os dados analisados são de um mês de aula, ocorrido de forma remota, no primeiro semestre de 2021, por 72 alunos de disciplinas de programação. Os alunos foram agrupados segundo o seu perfil Hexad e foi calculada a média de utilização de cada elemento por cada perfil de usuário.

Ao analisar a utilização real dos elementos de jogos, observou-se que os alunos com perfil Conquistador se interessaram pela maioria dos elementos de jogo, mas mostraram preferência pelas missões secundárias. O perfil Espírito Livre se mostrou compatível com os achados de Marczewski (2015), embora sejam necessárias novas análises para verificar se esse perfil está sendo beneficiado pelo estilo do jogo RPG. Esperava-se que o Filantropo fizesse mais uso de doação de itens, porém os elementos mais acessados por eles foram o easter eggs e escolha anarquista, fato que constituiu uma surpresa.

O perfil Jogador foi um dos perfis que mais explorou os elementos de jogos, tendo utilizado todos os 22 elementos implementados. Como era de se esperar, o perfil Jogador foi quem mais acessou o elemento infobox, que é um elemento de competição, pois permite que um jogador tenha acesso às conquistas de outro jogador. Como a informação é facilmente acessada no infobox, esta pode ser uma das razões para uma menor quantidade de acessos ao ranking. Por fim, o perfil Socializador teve preferência pela coleta de moedas e não pelo envio de mensagens para outros alunos. Será necessário um estudo mais aprofundado sobre o uso desse elemento, uma vez que o perfil jogador, que tende a buscar recompensa, foi quem mais enviou mensagens para outros alunos.

Há algumas divergências entre os achados deste artigo com outras pesquisas em relação às preferências dos usuários. No entanto, ainda não há unanimidade na literatura sobre quais são os elementos mais apreciados por cada perfil. Entre as razões para isso tem-se que grande parte das identificações dos perfis é baseada em questionários e não no comportamento do jogador. Além disso, a forma como são projetados esses elementos pode influenciar na escolha do jogador.

A principal contribuição deste trabalho são as análises de preferências dos jogadores com base na efetiva utilização dos elementos de jogos, além de oferecer um variado número de elementos de jogos e compor uma base de dados de utilização dos jogadores. Como trabalhos futuros, pretende-se fazer uma análise mais aprofundada das variáveis, adicionar novas variáveis, fazer uma análise qualitativa da utilização do jogo de forma a avaliar se o design do jogo pode estar interferindo na escolha dos alunos e analisar se o jogo ajudou a melhorar o engajamento, aproveitamento e índices de evasão.

\section{Agradecimentos}

Esta pesquisa, realizada no âmbito do Projeto Samsung-UFAM de Ensino e Pesquisa (SUPER), nos termos do artigo 48 do Decreto $n^{\circ}$ 6.008/2006 (SUFRAMA), foi parcialmente financiada pela Samsung Eletrônica da Amazônia Ltda., Nos termos da Lei Federal $\mathbf{n}^{\mathbf{o}}$ 8.387/1991, por meio dos convênios 001/2020 e 003/2019, firmados com a Universidade Federal do Amazonas e a FAEPI, Brasil. O presente trabalho foi realizado com apoio da Coordenação de Aperfeiçoamento de Pessoal de Nível Superior - Brasil (CAPES) Código de Financiamento 001. 


\section{Referências}

Amado, C. M. and Roleda, L. S. (2020). Game element preferences and engagement of different hexad player types in a gamified physics course. In Proceedings of the 2020 11th International Conference on E-Education, E-Business, E-Management, and E-Learning, pages 261-267.

Bartle, R. (1996). Hearts, clubs, diamonds, spades: Players who suit muds. Journal of MUD research, 1(1):19.

Bez, J. L., Tonin, N. A., and Rodegheri, P. R. (2014). Uri online judge academic: A tool for algorithms and programming classes. In 2014 9th International Conference on Computer Science \& Education, pages 149-152. IEEE.

Bovermann, K. and Bastiaens, T. J. (2020). Towards a motivational design? connecting gamification user types and online learning activities. Research and Practice in Technology Enhanced Learning, 15(1):1.

Galvão, L., Fernandes, D., and Gadelha, B. (2016). Juiz online como ferramenta de apoio a uma metodologia de ensino híbrido em programação. In Brazilian Symposium on Computers in Education (Simpósio Brasileiro de Informática na Educação-SBIE), volume 27, page 140 .

Hallifax, S., Serna, A., Marty, J.-C., and Lavoué, E. (2019). Adaptive gamification in education: A literature review of current trends and developments. In European conference on technology enhanced learning, pages 294-307. Springer.

Handelsman, M. M., Briggs, W. L., Sullivan, N., and Towler, A. (2005). A measure of college student course engagement. The Journal of Educational Research, 98(3):184192.

Hassan, M. A., Habiba, U., Majeed, F., and Shoaib, M. (2019). Adaptive gamification in e-learning based on students' learning styles. Interactive Learning Environments, pages $1-21$.

Jia, Y., Xu, B., Karanam, Y., and Voida, S. (2016). Personality-targeted gamification: a survey study on personality traits and motivational affordances. In Proceedings of the 2016 CHI Conference on Human Factors in Computing Systems, pages 2001-2013.

Kasahara, R., Sakamoto, K., Washizaki, H., and Fukazawa, Y. (2019). Applying gamification to motivate students to write high-quality code in programming assignments. In Proceedings of the 2019 ACM Conference on Innovation and Technology in Computer Science Education, pages 92-98.

Klock, A. C. T., Gasparini, I., Pimenta, M. S., and Hamari, J. (2020). Tailored gamification: A review of literature. International Journal of Human-Computer Studies, page 102495.

Kurnia, A., Lim, A., and Cheang, B. (2001). Online judge. Computers Education, 36(4):299-315.

Lopez, C. E. and Tucker, C. S. (2019). The effects of player type on performance: A gamification case study. Computers in Human Behavior, 91:333-345. 
Lowry, P. B., Gaskin, J., Twyman, N., Hammer, B., and Roberts, T. (2012). Taking 'fun and games' seriously: Proposing the hedonic-motivation system adoption model (hmsam). Journal of the association for information systems, 14(11):617-671.

Marczewski, A. (2015). Even ninja monkeys like to play. CreateSpace Indep. Publish Platform, Charleston, Chapter User Types, pages 69-84.

Melo, R., Pessoa, M., Haydar, G., de Oliveira, D. B. F., de Oliveira, E. H. T., de Carvalho, L. S. G., Conte, T., and Pereira, F. D. (2021). Um estudo sobre a relação entre os elementos de jogos e os tipos de usuários de sistemas gamificados. In Anais Estendidos do Simpósio Brasileiro de Educação em Computação, pages 35-36. SBC.

Nacke, L. E., Bateman, C., and Mandryk, R. L. (2014). Brainhex: A neurobiological gamer typology survey. Entertainment computing, 5(1):55-62.

Oyibo, K. and Vassileva, J. (2019). Investigation of persuasive system design predictors of competitive behavior in fitness application: A mixed-method approach. Digital health, 5:2055207619878601.

Pereira, F. D., Oliveira, E. H., Oliveira, D. B., Cristea, A. I., Carvalho, L. S., Fonseca, S. C., Toda, A., and Isotani, S. (2020). Using learning analytics in the amazonas: understanding students' behaviour in introductory programming. British Journal of Educational Technology.

Pessoa, M., Fernandes, D., de Carvalho, L. S. G., Oliveira, E., Nakamura, W., and Conte, T. (2019). Codeplay: Uma plataforma de gamificação baseada em jogos de rpg multiplayer. In Brazilian Symposium on Computers in Education (Simpósio Brasileiro de Informática na Educação-SBIE), volume 30, page 843.

Petit, J., Roura, S., Carmona, J., Cortadella, J., Duch, J., Gimnez, O., Mani, A., Mas, J., Rodrguez-Carbonell, E., Rubio, E., et al. (2017). Jutge. org: Characteristics and experiences. IEEE Transactions on Learning Technologies, 11(3):321-333.

Qian, Y. and Lehman, J. (2017). Students' misconceptions and other difficulties in introductory programming: A literature review. ACM Transactions on Computing Education (TOCE), 18(1):1-24.

Rodrigues, L., Toda, A. M., Palomino, P. T., Oliveira, W., and Isotani, S. (2020). Personalized gamification: A literature review of outcomes, experiments, and approaches. In Eighth International Conference on Technological Ecosystems for Enhancing Multiculturality, pages 699-706.

Ryan, R. M. and Deci, E. L. (2000a). Intrinsic and extrinsic motivations: Classic definitions and new directions. Contemporary educational psychology, 25(1):54-67.

Ryan, R. M. and Deci, E. L. (2000b). Self-determination theory and the facilitation of intrinsic motivation, social development, and well-being. American psychologist, 55(1):68.

Sinly, C., Rusli, A., and Winarno, P. (2018). Utilizing gamification to improve user participation in online judge. In 2018 Joint 10th International Conference on Soft Computing and Intelligent Systems (SCIS) and 19th International Symposium on Advanced Intelligent Systems (ISIS), pages 543-547. IEEE. 
Tondello, G. F., Arrambide, K., Ribeiro, G., Cen, A. J.-1., and Nacke, L. E. (2019a). "i don't fit into a single type": A trait model and scale of game playing preferences. In IFIP Conference on Human-Computer Interaction, pages 375-395. Springer.

Tondello, G. F., Mora, A., Marczewski, A., and Nacke, L. E. (2019b). Empirical validation of the gamification user types hexad scale in english and spanish. International Journal of Human-Computer Studies, 127:95-111.

Tondello, G. F., Wehbe, R. R., Diamond, L., Busch, M., Marczewski, A., and Nacke, L. E. (2016). The gamification user types hexad scale. In Proceedings of the 2016 annual symposium on computer-human interaction in play, pages 229-243. 